\title{
Brain Crosstalk with Other Organs in ICU Patient
}

\author{
Sai Saran ${ }^{1}$ Mohan Gurjar ${ }^{2}$ \\ ${ }^{1}$ Department of Critical Care Medicine, Super Speciality Cancer \\ Institute and Hospital, Lucknow, India \\ 2Department of Critical Care Medicine, Sanjay Gandhi Postgraduate \\ Institute of Medical Sciences (SGPGIMS), Lucknow, India
}

\begin{abstract}
Address for correspondence Mohan Gurjar, MD, PDCC, Department of Critical Care Medicine, Sanjay Gandhi Postgraduate Institute of Medical Sciences (SGPGIMS), Lucknow, 226014, India (e-mail:m.gurjar@rediffmail.com,m.gurjar@outlook.com).
\end{abstract}

\begin{abstract}
Crosstalk between various organs exists in the human body. This can be part of physiological reflexes such as cardiac reflexes that protect the organs during stressful stimuli or can be part of pathological conditions where an insult to an organ releases cytokines that cause distant effects on other organs. In critically ill patients, these crosstalks are independent of pre-existing common risk factors or the presence of new risk exposure during the treatment. Crosstalk can manifest in series or parallel. The human brain, being a control center of the human body, does crosstalk with almost every organ in the body. In this narrative review, crosstalk of the brain with various organs and sys-

Keywords

- brain

- crosstalk

- multi-organ failure tems such as the heart, lungs, liver, kidneys, gut, muscle, bone, skin, adipose tissue, and immune system is being discussed along with clinical manifestations and management. Future research might help to target these pathological processes in preventing progression of single-organ dysfunction to multi-organ failures in critically ill patients.
\end{abstract}

\section{Introduction}

Interorgan crosstalk plays crucial role in physiological and pathophysiological process. These crosstalk can be part of physiological reflexes such as baroreceptors, chemoreceptors, Cushing reflex, Bain-bridge reflex, and Bezold-Jarisch reflex which occur between the brain and heart, Hering-Breuer inflation reflex between the lungs and the brain, vomiting and vagovagal reflexes between the gut and the brain, reninangiotensin system between the brain, the lungs, the systemic vasculature, and the kidneys. ${ }^{1-6}$ This physiological crosstalk help in the maintenance of the body homeostasis, ${ }^{7}$ whereas other crosstalk might lead to pathogenesis, such as various syndromes including neurogenic stress, cardiomyopathy, and neurogenic pulmonary edema ${ }^{8-10}$

The pathological crosstalk can happen in "series" or "parallel" (—Fig. 1), leading to multiple organ dysfunction syndrome (MODS) in critically ill patients admitted to the intensive care unit (ICU). ${ }^{11}$ For example, serial organ failure can happen in conditions such as cardiogenic shock where massive myocardial infarction leads to ischemic hepatitis and acute kidney injury (AKI), whereas parallel organ failure is typically seen in septic shock where sepsis leads to acute respiratory distress syndrome (ARDS), AKI, sepsis-induced

received

August 4, 2019

accepted after revision

September 28, 2019

published online

December 18, 2019 cholestasis, and delirium. ${ }^{12,13}$ The pathophysiology behind this process is complicated and incompletely understood, which could be considered as "distant effects" of various cytokines released into the circulation due to events such as hypoperfusion, triggering interactions between immune cells of the human body, pathogen-associated molecular patterns (PAMPs), and damage-associated molecular patterns (DAMPs) released from the pathogen, leading to alteration in the body homeostasis. ${ }^{13}$ This crosstalk is beyond the presence of common risk factors for the brain and other organ dysfunctions such as age and coexisting comorbidities. The human brain, being a control center of the human body, does crosstalk with almost every organ and system in the body ( - Fig. 2).

We searched the PubMed database with terminologies such as "crosstalk," "brain," "heart," "lung," "liver," "kidney," "gut," "muscle," "bone," "skin," "immune system," "adipose," and "critically ill," for published articles between January 2000 and June 2019. From the relevant articles, cross-references were checked manually by both authors independently.

\section{Heart and Brain Crosstalk}

The link between the heart and the brain is practically seen in syncope or death after extreme emotions or stress, most
Copyright (O2019 Indian Society of Neuroanaesthesiology and Critical Care
License terms

() (1) $\ominus \circledast$
DOI https://doi.org/

10.1055/s-0039-3399474

ISSN 2348-0548.

.

(n)



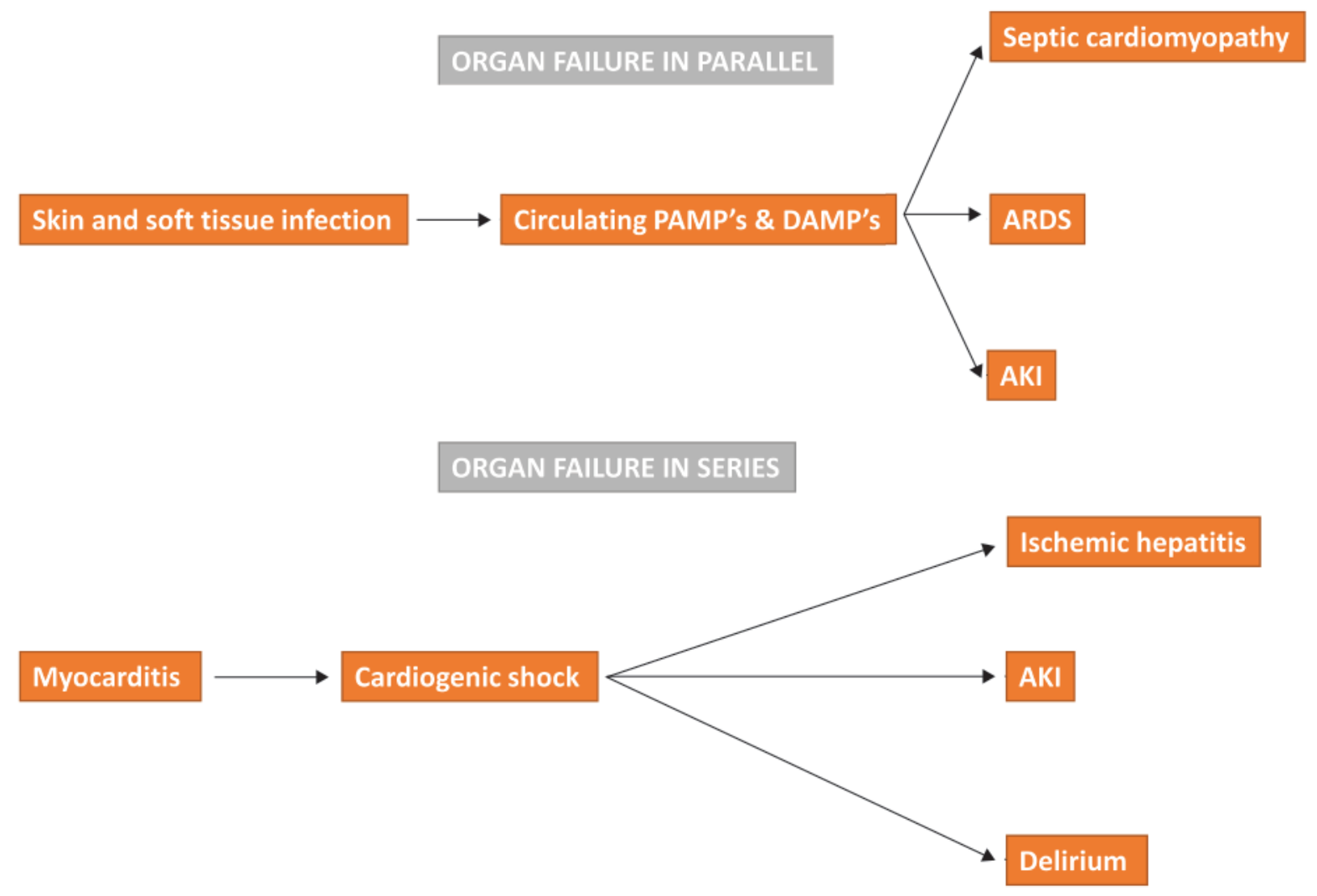

Fig. 1 Organ failure in "series" and "parallel." AKI, acute kidney injury; ARDS, acute respiratory distress syndrome; PAMP, pathogen-associated molecular patterns; DAMP, damage-associated molecular patterns.

likely due to heightened activation of the sympathetic nervous system. ${ }^{14}$ The effects of cardiovascular diseases on the nervous system, such as occurrence of cardioembolic stroke in patients with atrial fibrillation and neurocognitive decline in low cardiac output conditions, are well known. ${ }^{15}$ The other effect is the brain disorder affecting the heart, labeled as "neurogenic stress cardiomyopathy (NSC)" after sudden stressful events such as subarachnoid hemorrhage (SAH), traumatic brain injury (TBI), and epileptic seizures. ${ }^{16}$ This can manifest as arrhythmias, cardiogenic shock with electrocardiographic (ECG) changes such as ST-T wave abnormalities, and echocardiographic changes such as regional wall motion abnormalities, sometimes with typical apical and midventricular dysfunction manifesting as apical ballooning syndrome (Takotsubo cardiomyopathy). ${ }^{17,18}$ The pathophysiology behind this involves sympathetic activation following acute brain injury, leading to massive release of catecholamines leading to excessive $\beta_{1}$ and $\beta_{2}$ receptors stimulation along with reduced $\alpha_{2}$ modulation progressing to NSC. ${ }^{16}$ The brain centers involved in this cross-talk are cerebral cortex, amygdala, periaqueductal gray matter, locus coeruleus, hypothalamus, insula, rostral ventral lateral medulla and caudal ventrolateral medulla, nucleus tractus solitarii, and spinal lateral horn. ${ }^{16}$

Management of NSC, apart from resuscitation, involves judicious use of pharmacological measures such as $\beta$-blockers to inhibit the excessive upregulation of $\beta$-receptors apart from their potential role in protecting cardiac myocytes from excess catecholamine-induced apoptosis. Highly selective ultrashort acting $\beta$-blockers such as esmolol and landiolol have demonstrated adequate role in reducing the sympathetic drive by reducing the heart rate without affecting blood pressure. ${ }^{19}$ Inotropic drugs such as levosimendan, a calcium sensitizer, plays a role in such types of myocardial dysfunction without affecting heart rate and myocardial oxygen consumption..$^{20}$ This drug has also been implicated with potential benefits such as antiapoptotic and anti-stunning effects. ${ }^{21}$ In cases of persistent cardiogenic shock, requirement of intra-aortic balloon counter-pulsation therapy and ventricular support devices may be required. ${ }^{18}$ Drugs acting at central $\alpha_{2}$ receptor, such as dexmedetomidine targeting locus coeruleus, have a role in reducing the psychological stress by depressing the activity of amygdala, thereby preventing NSC. ${ }^{22}$

\section{Lung and Brain Crosstalk}

Neurocognitive problems in ARDS survivors definitely explain that there exists crosstalk between the brain and the lungs. ${ }^{23,24}$ Studies have shown that mechanical ventilation can induce an inflammatory response with distant organ failure, especially with the use of high tidal volume. ${ }^{25,26}$ The pathophysiology of this process starts with inflammation of alveoli, leading to diffuse alveolar injury, recruitment of neutrophils with cytokine release affecting the distal organs. Brain crosstalk with lungs is also being true with acute brain injury, leading to increase in pulmonary 


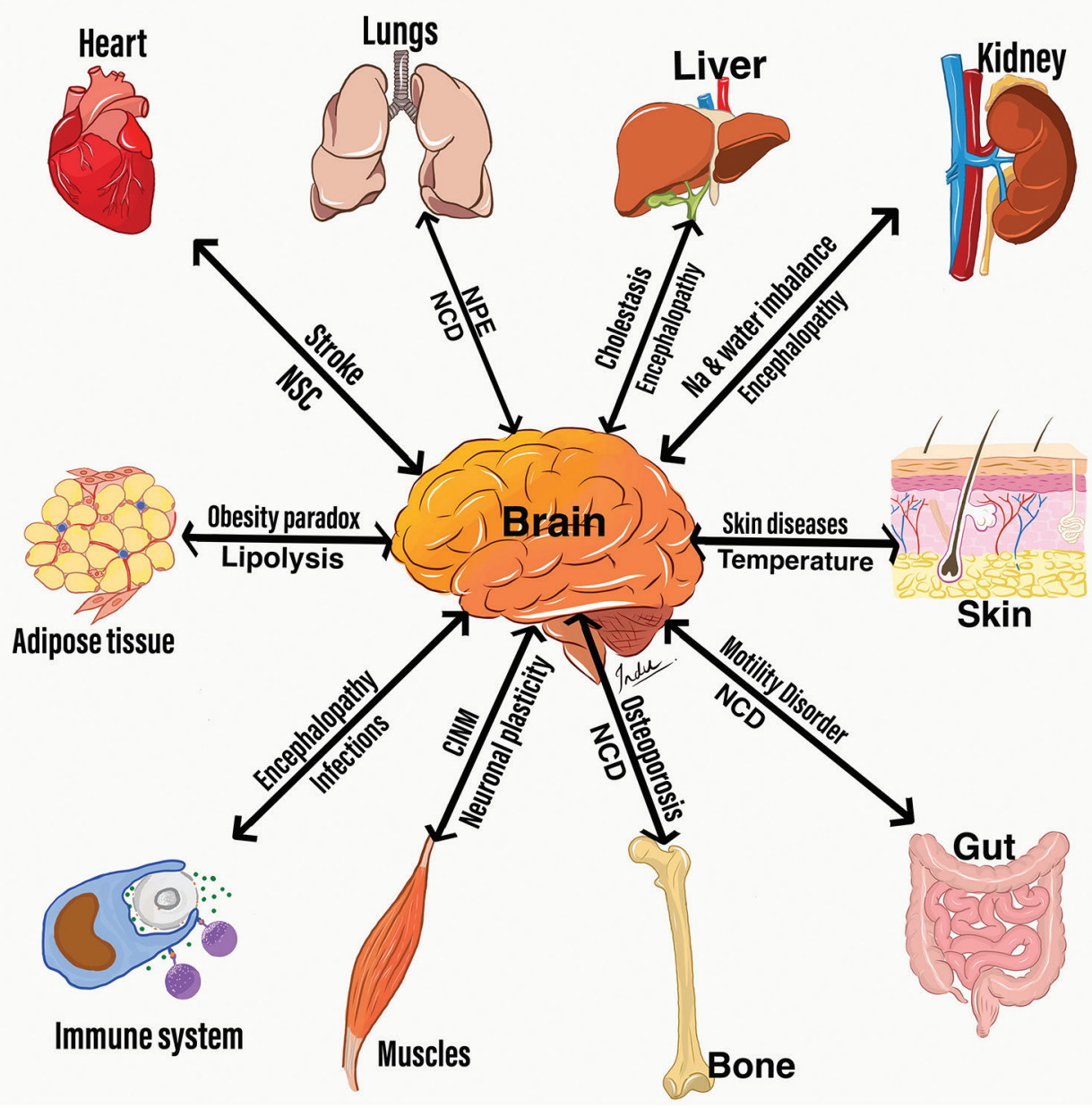

Fig. 2 Brain interacts with various organs and systems in critically ill patient. CINM, critical illness neuromyopathy; Na, sodium; NCD, neurocognitive dysfunction; NPE, neurogenic pulmonary edema; NSC, neurogenic stress cardiomyopathy.

complications in experimental settings. ${ }^{25}$ Various pulmonary complications that can occur after acute brain injury are neurogenic pulmonary edema (NPE); ventilation-perfusion mismatch, and structural parenchymal pathologies such as pneumonia, with increased predisposition to ventilator-associated pneumonia (VAP). ${ }^{27}$ Brain injury leads to sympathetic activation, releasing catecholamine manifesting as vasoconstriction (through increase in systemic and peripheral vascular resistance) that might cause endothelial damage and result in ARDS.

To prevent this pathological crosstalk, protective lung ventilation is required in patients with acute brain insult. Avoiding secondary insult (hypoxemia and hypotension) to the compromised brain forms the key in preventing this crosstalk. ${ }^{28}$ Rehabilitation therapies such as early mobilization, prevention, and early treatment of delirium in ICU may play a role in reducing the neurocognitive dysfunction (NCD) seen in patients with ARDS.

\section{Liver and Brain Crosstalk}

This can be explained through the well-known classic example of hepatic encephalopathy during decompensation of chronic liver disease and alcoholic liver disease. ${ }^{29}$ This crosstalk is linked through gut-related pathological changes such as increased permeability, altered microbiome of the gut, leading to "gut dysbiosis." ${ }^{30}$ The brain essentially requires glucose for its metabolism which is generated in high amounts by the liver. This process is linked to the lipid-sensing zone in the hypothalamus, where lipid oxidation takes place. Nucleus of the solitary tract and the dorsal motor vagal nucleus play key roles in this process. ${ }^{31}$

The other way of crosstalk is less known, that an insult to brain might lead to liver dysfunction. TBI leads to microglial activation in the pericontusional area, releasing various proinflammatory cytokines, leading to neutrophil and leukocyte recruitment not only in the brain but also in peripheral organs. Liver, being the most sensitive organs to inflammation and the storehouse of inflammatory molecules in the body, responds to this by releasing further chemokines. These chemokines further amplify the primary injury in the brain tissue by increasing the circulating immune cells. Serum amyloid A-1 (SAA-1), one of the well-studied chemokines released as part of an acute phase response, plays a key role in pathogenesis. ${ }^{31}$ Indeed, SAA- 1 has a role as a prognostic biomarker indicating the severity of brain injury. Drugs blocking SAA-1 synthesis are being studied to halt the liver failure that can occur following an acute brain insult. ${ }^{31}$ 


\section{Kidney and Brain Crosstalk}

Kidney failure leads to neurological issues such as cognitive decline, cerebrovascular events, peripheral neuropathy, dialysis disequilibrium syndrome, and even acute brain injury. ${ }^{32}$ These pathophysiological processes suggest that there exists a definitive crosstalk between these organs. ${ }^{33}$ This crosstalk can be mediated through hormones (antidiuretic hormone), alterations in the renin-angiotensin axis, and through pathological insults such as impaired blood-brain barrier integrity, alteration in catecholamine levels, water, acid-base and electrolyte disturbances, and toxin accumulation (uremic encephalopathy) and is labeled as "reno-cerebral reflex."33,34 On the other side, the brain insult could affect renal function which may manifest as hyponatremia (either syndrome of inappropriate antidiuretic hormone secretion [SIADH] or cerebral salt wasting [CSW]), and acute kidney injury (due to increased visceral sympathetic nervous system activation).

\section{Gut and Brain Crosstalk}

Alteration of gut microbiome has been implicated in many neurodegenerative diseases such as mood and depression disorders. ${ }^{35}$ The intestinal microbiota plays an important role in maintaining the intestinal barrier permeability. Wherever there is dysbiosis, gut-associated lymphoid tissue (GALT) gets activated and the permeability gets altered, leading to the spread of inflammatory cytokines to distant organs causing distant effects. Further, there exists anatomical resemblance between the gut barrier and the blood-brain barrier, both having epithelial cells, endothelial cells, and cellular tight junctions, which can lead to similar effects on the CNS. ${ }^{35}$ Gut microbiota gets extremely altered in critically ill patients due to various reasons such as administration of antibiotics, bowel rest, and feeding practices. This alteration of the gut microbiota might also be an explanation of various neurocognitive disorders that can happen during the ICU stay such as delirium and after discharge such as posttraumatic stress disorder (PTSD) and depression, which need further research. ${ }^{36}$ The other way of neurological insult causing gut dysfunction can easily be linked as most of the control centers for vomiting, peristalsis, and defecation are present in the brain.

\section{Muscle and Brain Crosstalk}

Skeletal muscle, the most abundant organ in the human body is affected in critically ill patients due to immobilization, leading to atrophy. There are other disorders linked to immobilization in the ICU, such as critical illness neuromyopathy (CINM). ${ }^{37}$ There exists crosstalk between the muscle, liver, and brain through "myokines," which exert autocrine, paracrine, and/or endocrine effects. Exercise leads to the release of L-lactate, $\beta$-hydroxybutyrate, irisin, and cathepsin-B, which play a role in hippocampal neurogenesis enhancing angiogenesis, neurogenesis, memory, sleep, and mood..$^{38}$ This provides a further insight into the role of early mobilization in the ICU to prevent delirium and other neurocognitive disorders.

\section{Bone and Brain Crosstalk}

Many patients with neurological disorders such as Alzheimer's disease, schizophrenia, and major depressive disorder develop osteoporosis, and patients with bone diseases such as cleidocranial dysplasia and osteopetrosis, develop cognitive decline with progression to mental retardation, suggesting that there exists certain crosstalk between these organs. ${ }^{39,40}$ Diseases such as osteoporosis create confusion whether the pathogenesis is at the brain or bone level. ${ }^{39}$ Bone, known for its skeletal framework in the human body, is an endocrine organ which talks with other organs and plays key role in homeostasis. This can happen through two well-studied molecules "fibroblast growth factor (FGF23)," regulating calcium and phosphorous metabolism, and "osteocalcin," playing a role in central neuronal regulation. Osteocalcin plays a role in crosstalk influencing brain development and cognitive function. ${ }^{41}$ This hormone can cross the blood-brain barrier and placenta, playing an important role in body homeostasis.

\section{Skin and Brain Crosstalk}

Acute stressful stimuli lead to the release of neurotrophins and neuropeptides from the central nervous system, resulting in inflammation in various organs including skin, which is likely to aggravate or trigger dermatological diseases. ${ }^{40}$ There is evidence that skin diseases such as atopic dermatitis, psoriasis, alopecia areata (AA), or chronic urticaria get aggravated during stress. The other way is well known where temperature changes in the skin (hypothermia or hyperthermia) can activate the hypothalamus and get the feedback stimulus, thereby maintaining body homeostasis. ${ }^{42}$

\section{Immune System and Brain Crosstalk}

It remains an unanswered question as to why patients with brain injury have high predisposition to various infections such as pneumonia, blood stream and other infections. Immune system communicates to the CNS through glial cells, especially microglia. Choroid plexus (CP), due to its location, structure, and immunological receptivity, forms the key interlink for this crosstalk, by performing as a gatekeeper to the immune cell trafficking into the CNS ${ }^{43}$ Inflammation activates microglia and the activated microglia play a role in enhancing innate immunity by upregulating cell surface receptors such as pattern recognition receptors (PRR).44,45 Neurons also participate in this neuroimmune crosstalk by controlling glial cells and infiltrated T cells. Neurons, glial cells, and immune cells act as a coordinated network to maintain body homeostasis. ${ }^{43}$ Neuropeptides, cytokines, and neurotransmitters released by both immune and nervous systems mediate this bidirectional communication. In conditions such as infection, the immune system gets activated which in turn changes the morphology, functioning of glia, and other immune cells of the brain that secrete high levels of proinflammatory cytokines, further enhancing the crosstalk. ${ }^{44}$ This pathophysiology can affect the memory, neural plasticity, and neurogenesis thereby manifesting as various 
types of encephalopathy, such as sepsis, for which clear pathogenesis is still unknown. ${ }^{46,47}$

\section{Adipose Tissue and Brain Crosstalk}

Ability to survive in metabolic challenges, such as temperature variations and starvation, comes from lipolysis. It is a known fact that obesity is associated with lower mortality in strokes and reduced risk of recurrence of stroke; this is known as "obesity paradox." 48 The levels of anti-inflammatory cytokines such as IL-10 released from adipose tissue were higher in obese patients than in nonobese patints. ${ }^{49}$ Apart from this, there exist specific central neural centers regulating adipose tissue depots. Leptin released from fat cells plays a role in thermoregulation and glucose homeostasis by acting on the hypothalamus, which acts as a neuroendocrine adaptation to fasting. ${ }^{50}$ Activation of the sympathetic system decreases the activity of brown adipose tissue and increases the activity (lipolysis) of white adipose tissue, leading to fatty acid mobilization. Treatment with catecholamine (norepinephrine) reduces leptin levels, and can therefore predispose the patients to temperature fluctuations and hypoglycemia. ${ }^{50}$

\section{Conclusion}

As the brain harbors almost all the control centers of each organ in the human body, various pathogenic process such as neurogenic stress cardiomyopathy, ventilator-induced lung injury, neurocognitive deficits in ARDS survivors, encephalopathy in renal and liver failures, and critical illness neuromyopathy exist in which crosstalk plays a role in pathogenesis. In future, research might help to target this pathological crosstalk at an early stage to prevent progression of single-organ dysfunction to multiorgan failures that can occur in critically ill patients.

\section{Conflict of Interest}

None declared.

\section{Acknowledgment}

We are thankful to Dr. Indubala Maurya (Assistant Professor, Department of Oncoanesthesia, Superspeciality Cancer Institute and Hospital, Lucknow) for her artwork for - Fig. 2.

\section{References}

1 Kociol R, Rogers J, Shaw A. Organ cross talk in the critically ill: the heart and kidney. Blood Purif 2009;27(4):311-320

2 Crystal GJ, Salem MR. The Bainbridge and the "reverse" Bainbridge reflexes: history, physiology, and clinical relevance. Anesth Analg 2012;114(3):520-532

3 Campagna JA, Carter C. Clinical relevance of the Bezold-Jarisch reflex. Anesthesiology 2003;98(5):1250-1260

4 Dutschmann M, Bautista TG, Mörschel M, Dick TE. Learning to breathe: habituation of Hering-Breuer inflation reflex emerges with postnatal brainstem maturation. Respir Physiol Neurobiol 2014;195:44-49

5 Browning KN, Travagli RA. Central nervous system control of gastrointestinal motility and secretion and modulation of gastrointestinal functions. Compr Physiol 2014;4(4):1339-1368
6 Sparks MA, Crowley SD, Gurley SB, Mirotsou M, Coffman TM. Classical renin-angiotensin system in kidney physiology. Compr Physiol 2014;4(3):1201-1228

7 Jenie IM. Cardiovascular reflexes. Mutiara Medika 2007;1(7):43-50

8 Cruz DN. Cardiorenal syndrome in critical care: the acute cardiorenal and renocardiac syndromes. Adv Chronic Kidney Dis 2013;20(1):56-66

9 Francoz C, Durand F, Kahn JA, Genyk YS, Nadim MK. Hepatorenal syndrome. Clin J Am Soc Nephrol 2019;14(5):774-781

10 Papiris SA, Manali ED, Kalomenidis I, Kapotsis GE, Karakatsani A, Roussos C. Bench-to-bedside review: pulmonary-renal syndromes-an update for the intensivist. Crit Care 2007;11(3):213

11 Armutcu F. Organ crosstalk: the potent roles of inflammation and fibrotic changes in the course of organ interactions. Inflamm Res 2019;68(10):825-839

12 Ciobanu AO, Gherasim L. Ischemic hepatitis-intercorrelated pathology. Maedica (Buchar) 2018;13(1):5-11

13 Bauer M, Coldewey SM, Leitner M, Löffler B, Weis S, Wetzker R. Deterioration of organ function as a hallmark in sepsis: the cellular perspective. Front Immunol 2018;9(9):1460

14 Tahsili-Fahadan P, Geocadin RG. Heart-brain axis. Circ Res 2017;120(3):559-572

15 Finsterer J, Stöllberger C. Neurological complications of cardiac disease (heart brain disorders). Minerva Med 2016;107(1):14-25

16 Mazzeo AT, Micalizzi A, Mascia L, Scicolone A, Siracusano L. Brain-heart crosstalk: the many faces of stress-related cardiomyopathy syndromes in anaesthesia and intensive care. $\mathrm{Br}$ Anaesth 2014;112(5):803-815

17 Guglin M, Novotorova I. Neurogenic stunned myocardium and takotsubo cardiomyopathy are the same syndrome: a pooled analysis. Congest Heart Fail 2011;17(3):127-132

18 Ripoll JG, Blackshear JL, Díaz-Gómez JL. Acute cardiac complications in critical brain disease. Neurosurg Clin N Am 2018;29(2):281-297

19 Kawaguchi M, Utada K, Yoshitani K, et al; Intraoperative Landiolol for Intracranial Aneurysm Surgery Trial (ILAST) Investigators. Effects of a short-acting $[\beta] 1$ receptor antagonist landiolol on hemodynamics and tissue injury markers in patients with subarachnoid hemorrhage undergoing intracranial aneurysm surgery. J Neurosurg Anesthesiol 2010;22(3):230-239

20 Padayachee L. Levosimendan: the inotrope of choice in cardiogenic shock secondary to takotsubo cardiomyopathy? Heart Lung Circ 2007;16(Suppl 3):S65-S70

21 Paraskevaidis IA, Parissis JT, Th Kremastinos D. Antiinflammatory and anti-apoptotic effects of levosimendan in decompensated heart failure: a novel mechanism of drug-induced improvement in contractile performance of the failing heart. Curr Med Chem Cardiovasc Hematol Agents 2005;3(3):243-247

22 Ali A, Ahmad MQ Malik MB, et al. Retraction: neurogenic stunned myocardium: a literature review. Cureus 2019;11(4):r12

23 Han DW. Brain and lung: dangerous crosstalk. Korean J Anesthesiol 2017;70(2):116-117

24 Gonzalvo R, Martí-Sistac O, Blanch L, López-Aguilar J. Benchto-bedside review: brain-lung interaction in the critically ill-a pending issue revisited. Crit Care 2007;11(3):216

25 Heuer JF, Pelosi P, Hermann P, et al. Acute effects of intracranial hypertension and ARDS on pulmonary and neuronal damage: a randomized experimental study in pigs. Intensive Care Med 2011;37(7):1182-1191

26 Quilez ME, Fuster G, Villar J, et al. Injurious mechanical ventilation affects neuronal activation in ventilated rats. Crit Care 2011;15(3):R124 
27 Pelosi P, Rocco PR. The lung and the brain: a dangerous crosstalk. Crit Care 2011;15(3):168

28 Blanch L, Quintel M. Lung-brain cross talk in the critically ill. Intensive Care Med 2017;43(4):557-559

29 Poole LG, Dolin CE, Arteel GE. Organ-organ crosstalk and alcoholic liver disease. Biomolecules 2017;7(3):1-16

30 Pocai A, Obici S, Schwartz GJ, Rossetti L. A brain-liver circuit regulates glucose homeostasis. Cell Metab 2005;1(1):53-61

31 Villapol S. Consequences of hepatic damage after traumatic brain injury: current outlook and potential therapeutic targets. Neural Regen Res 2016;11(2):226-227

32 Li X, Hassoun HT, Santora R, Rabb H. Organ crosstalk: the role of the kidney. Curr Opin Crit Care 2009;15(6):481-487

33 Afsar B, Sag AA, Yalcin CE, et al. Brain-kidney crosstalk: definition and emerging evidence. Eur J Intern Med 2016;36:7-12

34 Cao W, Li A, Li J, et al. Reno-cerebral reflex activates the renin-angiotensin system, promoting oxidative stress and renal damage after ischemia-reperfusion injury. Antioxid Redox Signal 2017;27(7):415-432

35 Luczynski P, McVey Neufeld KA, Oriach CS, Clarke G, Dinan TG, Cryan JF. Growing up in a bubble: using germ-free animals to assess the influence of the gut microbiota on brain and behavior. Int J Neuropsychopharmacol 2016;19(8):pyw020

36 Cenit MC, Sanz Y, Codoñer-Franch P. Influence of gut microbiota on neuropsychiatric disorders. World J Gastroenterol 2017;23(30):5486-5498

37 Shepherd S, Batra A, Lerner DP. Review of critical illness myopathy and neuropathy. Neurohospitalist 2017;7(1):41-48

38 Delezie J, Handschin C. Endocrine crosstalk between skeletal muscle and the brain. Front Neurol 2018;9:698

39 Birge SJ. Osteoporotic fractures: a brain or bone disease? Curr Osteoporos Rep 2008;6(2):57-61
40 Joachim RA, Kuhlmei A, Dinh QT, et al. Neuronal plasticity of the "brain-skin connection": stress-triggered up-regulation of neuropeptides in dorsal root ganglia and skin via nerve growth factor-dependent pathways. J Mol Med (Berl) 2007;85 (12):1369-1378

41 Rousseaud A, Moriceau S, Ramos-Brossier M, Oury F. Bonebrain crosstalk and potential associated diseases. Horm Mol Biol Clin Investig 2016;28(2):69-83

42 Morrison SF. Central control of body temperature. F1000 Res 2016;5(5):880

43 Schwartz M, Baruch K. The resolution of neuroinflammation in neurodegeneration: leukocyte recruitment via the choroid plexus. EMBO J 2014;33(1):7-22

44 Tian L, Ma L, Kaarela T, Li Z. Neuroimmune crosstalk in the central nervous system and its significance for neurological diseases. J Neuroinflammation 2012;9(1):155

45 Schwartz M, Deczkowska A. Neurological disease as a failure of brain-immune crosstalk: the multiple faces of neuroinflammation. Trends Immunol 2016;37(10):668-679

46 Chaudhry N, Duggal AK. Sepsis associated encephalopathy. Adv Med 2014;2014:762320

47 Heming N, Mazeraud A, Verdonk F, Bozza FA, Chrétien F, Sharshar T. Neuroanatomy of sepsis-associated encephalopathy. Crit Care 2017;21(1):65

48 Andersen KK, Olsen TS. The obesity paradox in stroke: lower mortality and lower risk of readmission for recurrent stroke in obese stroke patients. Int J Stroke 2015;10(1):99-104

49 Rodríguez-Castro E, Rodríguez-Yáñez $M$, Arias-Rivas S, et al. Obesity paradox in ischemic stroke: clinical and molecular insights. Transl Stroke Res 2019; doi: 10.1007/ s12975-019-00695-x

50 Caron A, Lee S, Elmquist JK, Gautron L. Leptin and brainadipose crosstalks. Nat Rev Neurosci 2018;19(3):153-165 